\title{
Ultrastructure and Enzymatic Deficiency of Fibroblast Cultures in Type II Glycogenosis
}

\author{
George Hug ${ }^{[20]}$, William K. Schubert, and Shirley Soukup \\ The Children's Hospital Research Foundation and the Department of Pediatrics, University of Cincinnati \\ College of Medicine, Cincinnati, Ohio, USA
}

\begin{abstract}
Extract
Fibroblast cultures from skin biopsy specimens of a girl with type II glycogenosis showed less than $5 \%$ of control activity of lysosomal acid $\alpha$-glucosidase. These cells showed numerous intracellular vacuoles that resembled the abnormal lysosomes found in liver and other tissues of the patient. Their characteristic ultrastructural abnormality was seen in fibroblast and epithelial cells of the primary cultures and thereafter in all the subcultures of which to date eight passages have been examined. Control fibroblast cultures did not contain such inclusions.
\end{abstract}

\section{Speculation}

Demonstration of the disease-specific ultrastructural abnormality indicates the usefulness of fibroblast cultures to study pathophysiology and potential treatment in type II glycogenosis. In addition, fibroblast cultures of other lysosomal diseases may serve a similar purpose.

\section{Introduction}

Type II glycogenosis is a hereditary metabolic disease of infancy. The majority of affected individuals develop muscular weakness and progressive enlargement of the heart and die of cardiac failure [2]. Biochemically, the disease is associated with deficient activity of lysosomal acid $\alpha$-1,4-glucan glucohydrolase $(\alpha$-glucosidase, EC.3.2.1.20) and with increased concentrations of glycogen in all tissues $[4,5]$. Numerous membrane-bounded accumulations of glycogen (presumably abnormal lysosomes) were present in electron photomicrographs of liver [1]. Similar structures have been seen in less abundance in other tissues [5].

Detailed study of disease mechanism and design of effective treatment are difficult to accomplish for lack of a suitable experimental system. Electron microscopic examination of fibroblast cultures may provide this system.

\section{Patients and Methods}

Skin biopsies were performed in an 8-month-old white girl [15] with the clinical, biochemical, and ultrastructural features of type II glycogenosis (Table I, Fig. 1). Primary fibroblast cultures were obtained from multiple skin explants and were grown on slides in Leighton tubes. The cell culture medium was a $1: 1$ mixture of Eagle's TEM and medium 199 (1 ml per tube) supplemented with $20 \%$ bovine serum, streptomycin, and penicillin (100 units/ml of each). Medium was changed three times per week. The cells were subcultured into other Leighton tubes with slides and into T-flasks or prescription bottles. Cultures were processed 


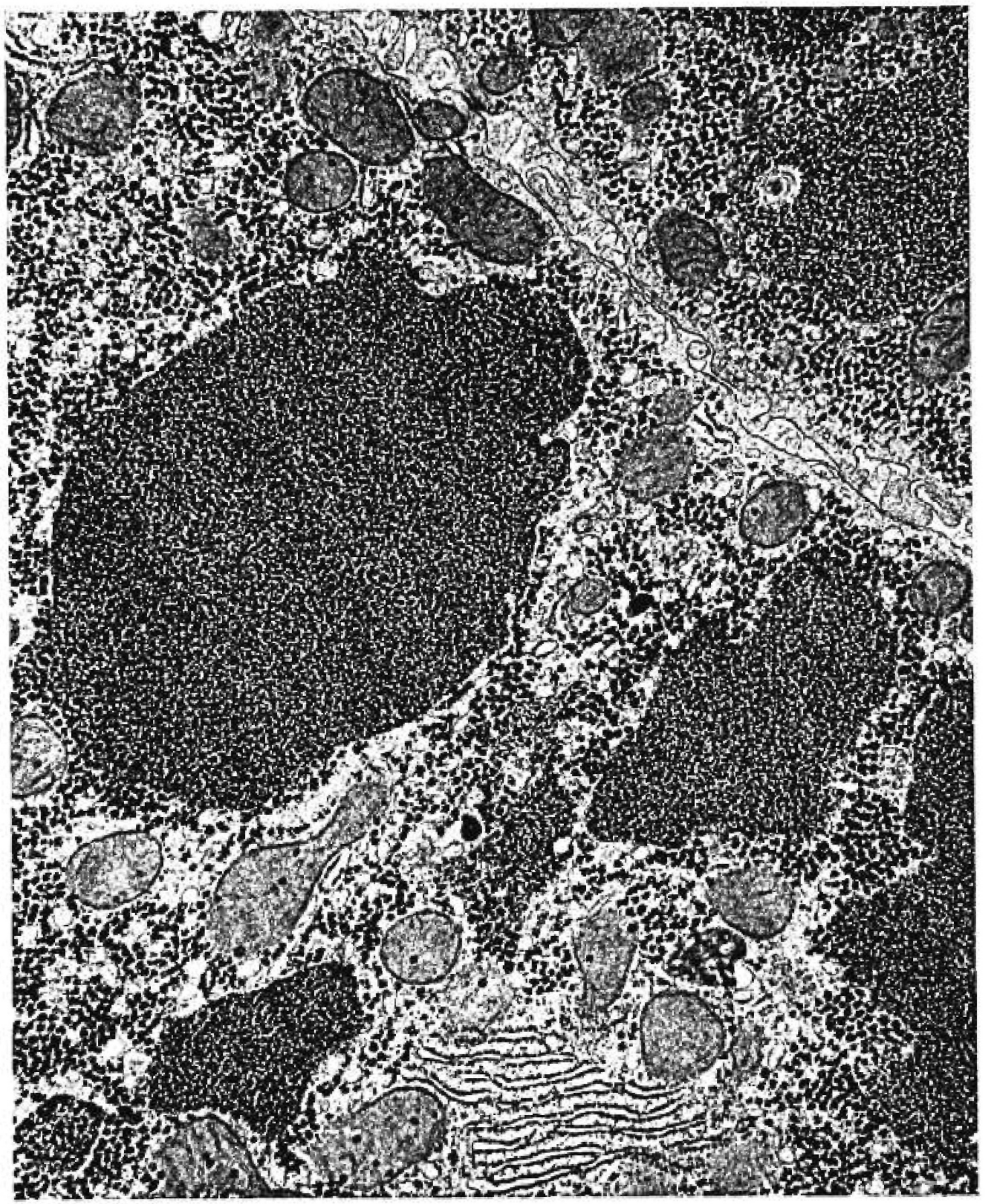

Fig. 1. Patient's liver biopsy specimen. There are parts of two hepatocytes that appear normal except for membrane-bounded

for the first time 3-8 weeks after initiation for determination of $\alpha$-glucosidase activity and for electron microscopy, as well as for continuation as subcultures. Further passages were at weekly intervals. vacuoles (abnormal lysosomes). The vacuoles differ in size and shape. They are packed with glycogen particles. $\times 16,000$.

For determination of $\alpha$-glucosidase activity the cultures were trypsinized and washed three times in Hank's balanced salt solution. Subsequent homogenization in sodium acetate buffer $0.02 \mathrm{M}, \mathrm{pH} 4.0$, released 